\title{
AXL receptor tyrosine kinase is increased in patients with
}

\section{heart failure}

M. Batlle $\mathrm{PhD}^{1 *}$, P. Recarte-Pelz $\mathrm{MSc}^{2}$, E. Roig MD, $\mathrm{PhD}^{3}, \mathrm{MA}$ Castel MD, $\mathrm{PhD}^{4}, \mathrm{M}$.

Cardona $\mathrm{MD}^{5}$, M. Farrero $\mathrm{MD}^{6}$, J. Ortiz $\mathrm{MD}^{7}, \mathrm{PhD}$, B. Campos $\mathrm{PhD}^{8}$, M.J. Pulgarín

$\mathrm{BSc}^{9}$, J. Ramírez $\mathrm{MD}^{10}$, PhD, F. Pérez-Villa MD, $\mathrm{PhD}^{11 \S}$, P. García de Frutos $\mathrm{PhD}^{12 \S}$

${ }^{1}$ Researcher at Institute of Biomedical Research August Pi i Sunyer (IDIBAPS) and the Thorax Institute, Cardiology Department, Hospital Clínic de Barcelona. This author takes responsibility for all aspects of the reliability and freedom from bias of the data presented and their discussed interpretation.

${ }^{2} \mathrm{Ph} . \mathrm{D}$. Student at the Department of Cell Death and Proliferation at Institut $\mathrm{d}$ 'Investigacions Biomèdiques de Barcelona (IIBB-CSIC) and IDIBAPS. This author takes responsibility for all aspects of the reliability and freedom from bias of the data presented and their discussed interpretation.

${ }^{3}$ Head of Heart Failure Unit at the Cardiology Department, Hospital de la Santa Creu i Sant Pau, Institut de Recerca Biomèdica (IIB Sant Pau). Universitat Autònoma de Barcelona. This author takes responsibility for all aspects of the reliability and freedom from bias of the data presented and their discussed interpretation.

${ }^{4}$ Cardiologist at the Thorax Institute, Cardiology Department, Hospital Clínic de Barcelona and researcher at Institute of Biomedical Research August Pi i Sunyer (IDIBAPS). This author takes responsibility for all aspects of the reliability and freedom from bias of the data presented and their discussed interpretation.

${ }^{5}$ Cardiologist at the Thorax Institute, Cardiology Department, Hospital Clínic de Barcelona and researcher at Institute of Biomedical Research August Pi i Sunyer (IDIBAPS). This author takes responsibility for all aspects of the reliability and freedom from bias of the data presented and their discussed interpretation.

${ }^{6}$ Cardiologist at the Thorax Institute, Cardiology Department, Hospital Clínic de Barcelona and researcher at Institute of Biomedical Research August Pi i Sunyer (IDIBAPS). This author takes responsibility for all aspects of the reliability and freedom from bias of the data presented and their discussed interpretation.

${ }^{7}$ Cardiologist at the Thorax Institute, Cardiology Department, Hospital Clínic de Barcelona and researcher at Institute of Biomedical Research August Pi i Sunyer (IDIBAPS). This author takes responsibility for all aspects of the reliability and freedom from bias of the data presented and their discussed interpretation.

${ }^{8}$ Associate Professor at the Department of Public Health, Universitat de Barcelona, Spain. This author takes responsibility for all aspects of the reliability and freedom from bias of the data presented and their discussed interpretation.

${ }^{9}$ Researcher at Institute of Biomedical Research August Pi i Sunyer (IDIBAPS) and the Thorax Institute, Cardiology Department, Hospital Clínic de Barcelona. This author takes responsibility for all aspects of the reliability and freedom from bias of the data presented and their discussed interpretation.

${ }^{10} \mathrm{Head}$ of the Pathological Anatomy Department, Hospital Clínic de Barcelona, Barcelona, Spain. This author takes responsibility for all aspects of the reliability and freedom from bias of the data presented and their discussed interpretation. 
${ }^{11}$ Head of the Heart Failure and Trasplant Unit at the Thorax Institute, Cardiology Department, Hospital Clínic de Barcelona and researcher at Institute of Biomedical Research August Pi i Sunyer (IDIBAPS). This author takes responsibility for all aspects of the reliability and freedom from bias of the data presented and their discussed interpretation.

${ }^{12} \mathrm{Head}$ of the Department of Cell Death and Proliferation at Institut d'Investigacions Biomèdiques de Barcelona (IIBB-CSIC) and IDIBAPS. This author takes responsibility for all aspects of the reliability and freedom from bias of the data presented and their discussed interpretation.

*, ${ }^{\S}$ These authors contributed equally to this work.

This work was performed at the IDIBAPS, at the Thorax Institute of Hospital Clínic de Barcelona and at the IIBB-CSIC.

Corresponding author; Montserrat Batlle, Ph.D., E-mail mbatlle@ clinic.cat, telephone +34932275400/3356, FAX +34932279305.

\section{Funding}

This work was supported by grants from the Fundació la Marató de TV3 2008 [project 081010, project 080121] and from the Spanish Network on Heart Failure REDINSCOR [V-2006-RET0308-O] and Red de Investigaciones Cardiovasculares RIC, by the Instituto de Salud Carlos III, Ministerio de Sanidad y Consumo.

Conflict of Interest: none declared

Keywords Heart failure; AXL receptor tyrosine kinase; prognosis, myocardial damage. 


\section{ABSTRACT}

Background. AXL is a membrane receptor tyrosine kinase highly expressed in the heart and has a conspicuous role in cardiovascular physiology. The role of AXL in heart failure (HF) has not been previously addressed.

Methods and Results. AXL protein was enhanced 6-fold in myocardial biopsies of end-stage HF patients undergoing heart transplantation compared to controls from heart donors $(P<0.0001)$. ADAM 10?. Next, we performed a transversal study of patients with chronic HF $(n=192)$ and a group of controls with no HF ( $n=67)$. sAXL and BNP circulating levels were quantified and clinical and demographic data were collected.

sAXL levels in serum were higher in HF $(86.3 \pm 2.0 \mathrm{ng} / \mathrm{mL})$ than in controls $(67.8 \pm 2.0$ ng/mL; $P<0.0001)$. Also, sAXL correlated with several parameters associated with worse prognosis in HF. Linear regression analysis indicated that serum creatinine, systolic blood pressure and atrial fibrillation, but not BNP levels, were predictive of sAXL levels. Cox regression analysis indicated that high sAXL values at enrollment time were related to the major HF events (all-cause mortality, heart transplantation and HF hospitalizations) at one year follow-up $(P<0.001)$, adding predictive value to high BNP levels.

Conclusions. Myocardial expression and serum concentration of AXL is elevated in HF patients compared to controls. Furthermore, peripheral sAXL correlates with parameters associated with the progression of HF and with HF events at short term follow-up. All together these results suggest that sAXL could belong to a new molecular pathway involved in myocardial damage in HF, independent from BNP. 


\section{Introduction}

Heart Failure (HF), characterized by a ventricular systolic or diastolic dysfunction, constitutes the end-stage of many heart diseases. Population prevalence of $\mathrm{HF}$ averages 2 to $3 \%$ increasing to $\geq 10 \%$ at 70 years of age or older. $(1,2)$ Epidemiological studies report a link between HF and low survival rates.(3) Several etiologies underlie the diagnosis of $\mathrm{HF}$, including pathological remodeling of the myocardium after an infarct. Other important etiologies include cardiomyopathies, hypertension, valvular heart disease, and toxins. Therefore, we can consider HF as a multifactorial syndrome with abnormalities of cell signaling, that are reflected in the contractile function of the myocardium as well as in cardiac cell survival and death. (4, 5)

Receptor tyrosine kinases (RTKs) are membrane proteins recognizing extracellular signals leading to cellular responses such as proliferation, arrest or activation. AXL belongs to the Tyro3, Axl and MerTK (TAM) subfamily, of RTKs and is a protein of particular interest as a potential player in the pathophysiology of the failing heart. Although the TAM receptors were first cloned as orphan receptors, affinity purification techniques led to the identification of Growth Arrest-Specific 6 protein (GAS6) as the AXL ligand.(6) GAS6 has significant structure and sequence similarity with the vitamin K-dependent protein S, but lacks its anticoagulant activity.(7) Cells of the vasculature including endothelial cells, vascular smooth muscle cells and fibroblasts synthesize and express GAS6 and AXL. $(8,9)$ According to the GeneAtlas data set, AXL is highly expressed in the heart, including cardiomyocytes, among other organs.(10) Membrane bound AXL can be shed by the effects of a Disintegrin and Metalloproteinase domain-containing protease (ADAM), ADAM10? leading to a 
soluble form that is detected in plasma as a stable complex with its ligand GAS6.(11, 12)

The aim of this project was to analyze the AXL myocardial and serum levels in patients with HF and whether it could be useful in assessing the stage of the HF. Hence, we analyzed the relationship of sAXL with parameters that are affected in HF and estimated the value of sAXL in predicting events at one year follow-up.

\section{Methods}

\section{Collection and analysis of human heart samples}

Human heart samples were analyzed in 2 populations: control samples $(n=11)$ were non-diseased biopsies. These samples were from organ donors whose heart could not be used for transplantation because they did not match the requirements of the potential heart receptors. Left ventricle samples were collected at the time of organ donation.

Case samples $(n=15)$ were pathological human left ventricle biopsies from patients with severe HF obtained at the time of cardiac transplantation, as described previously.(13) Whenever it was possible, sample harvest of around $1 \mathrm{~cm}^{3}$ myocardial biopsies was performed in a middle region between the apex and the base. The myocardial tissue was immediately immersed in liquid nitrogen. The Ethical Committee of the affiliated institution approved the protocol.

\section{Western Blot analysis of SAXL protein}

Myocardial proteins were extracted from left ventricle biopsies, if possible from the anterior wall. Samples were submerged in $1 \mathrm{~mL}$ of ice-cold protein lysis buffer containing: 50 mM Tris $\mathrm{HCl}$ pH 7.5 (T5941, Sigma), 150 mM NaCl (1.06404.5000, 
Merck), $1 \%$ Nonidet P-40 (Tergitol solution, NP40S, Sigma), 0.5\% sodium deoxycholate (D6750, Sigma), 0.1\% sodium dodecyl sulfate (L5750, Sigma), $1 \mathrm{mM}$ phenylmethanesulfonyl fluoride (P7626, Sigma), $1 \mathrm{mM}$ sodium orthovanadate (S6508, Sigma), $1 \mathrm{mM}$ Pefabloc (11429868001, Roche) and complete Mini Protease Inhibitor Cocktail (11836153001, Roche). Samples were homogenized with an Omni TH homogenizer (Omni International Inc.). After 1 hour of rotation at $4{ }^{\circ} \mathrm{C}$, samples were centrifuged at $10.000 \mathrm{~g}$ at $4{ }^{\circ} \mathrm{C}$ for 30 minutes. The upper phase was collected and the total protein concentration was quantified with the Pierce BCA protein Assay method (23227, Thermo Scientific, Pierce) relative to a BSA standard curve. Thirty $\mu \mathrm{g}$ of total protein extract were loaded to NuPage ${ }^{\circledR} 4-12 \%$ Bis-Tris Gel (NP0322) and a western blot was performed with the Novex® gels methodology (Invitrogen). Proteins were transferred from the gel to a nitrocellulose membrane using a blot gel transfer (IB301001) and the iBlot ${ }^{\circledR}$ Dry Blotting System. After 1 hour blockade of the membrane with phosphate buffered saline solution (PBS, Fisher Scientific), 0.1\% Tween 20 (P1379, Sigma-Aldrich) and 5\% of skimmed milk, it was incubated overnight at $4{ }^{\circ} \mathrm{C}$ with the AXL (c-20) primary antibody against the carboxy terminal protein of the full-length human protein diluted 1/1000 (sc-1096, Santa Cruz Biotechnology). Afterwards, the membrane was incubated during 1 hour with an HRP-Rabbit anti-Goat secondary antibody diluted 1/5000 (31402 Thermo Scientific). Final detection of the AXL protein band was accomplished with the ECL kit Supersignal West Pico Chemioluminescent Substrate (34080, Thermo Scientific).

Quantification of the integrated density of the bands around $150 \mathrm{kDa}$ was done with the ImageJ program (NIH, Maryland, USA). Each blot was loaded with $30 \mu \mathrm{g}$ of a standard sample of human myocardial protein for calibration between blots. Results are 
given in relative units (RU) as the ratio between the integrated densities of each sample divided by the integrated density of the standard.. ADAM10?

\section{Patient enrolment and collection of clinical data}

This was a transversal study of patients treated in a specialized outpatient $\mathrm{HF}$ clinic with chronic, stable HF with systolic dysfunction, characterized by an ejection fraction (EF) less or equal to $40 \%$ (mean evolution HF time of $7.5 \pm 0.6$ years). Participants were recruited in a consecutive manner based on presenting the inclusion criteria from May 2009 to November 2011 in the Hospital Clinic of Barcelona and from November 2010 to October 2011 in the Hospital Sant Pau of Barcelona. Patients belonged to New York Heart Association (NYHA) functional classes II, III or IV (mainly II and III). HF patients were diagnosed and assigned to a NYHA class by a group of cardiologists specialized in heart failure and heart transplantation according to the European Society of Cardiology criteria.(2) These patients were considered to have stable HF because they did not have a hospital admission with a diagnosis of HF during the previous month before enrolment.

Historical records from these patients were retrieved and an echocardiography taken within a year from the inclusion day was used for each patient. Clinical data such as ECG data, HF evolution time, functional capacity measured as 6-minute walking test (6MWT), cardiovascular risk factors (hypertension, dyslipidemia, diabetes mellitus and smoking), laboratory parameters and medication were entered for analysis. Ventricular dilation parameters, systolic function and the EF were assessed by echocardiography. One hundred ninety-two patients were included with a major reduced left ventricular function, and were designated as HF. Patients under 18 years of age were excluded as well as patients affected by other fibroproliferative diseases such as; kidney failure 
(creatinine levels> $3 \mathrm{mg} / \mathrm{dL}$ ), cirrhosis, bone metabolic disease, hyperthyroidism, pulmonary fibrosis, systemic sclerosis, macular degeneration and amyloidosis.

This study complies with the Declaration of Helsinki, the Ethical Committee of the affiliated institution approved the protocol and all subjects gave written, informed consent to participate in this study.

\section{Patient short term follow-up}

Follow-up data of one year $+/-1$ month were obtained from either a clinical or a telephone interview or from the clinical records. The events that were recorded were as follows: hospitalizations for either $\mathrm{HF}$, acute coronary syndrome, cardiorespiratory arrest, ventricular tachycardia or fibrillation; device implantation of either a pacemaker, an implantable cardioverter-defibrillator (ICD), cardiac resynchronization therapy (CRT with or without ICD); percutaneous revascularization; cardiac surgery (for either heart transplantation, coronary revascularization or others); ictus and all-cause mortality (cardiac and non-cardiac deaths).

\section{Controls characteristics}

Controls were a group of subjects with no HF with a lower proportion of male subjects $(46 \%)$ than the patients $(85 \%, P<0.0001)$. Controls mean age was $48.6 \pm 2.0$ years $(n=67)$ and was lower than the mean age of the patients $61.8 \pm 0.8$ years $(n=192$, $P<0.0001)$. HF patients had higher proportions of all risk factors than controls;

Hypertension $P<0.0001$, Dyslipidemia $P<0.0001$, Diabetes Mellitus $\quad P<0.01$, Current/former smoker $P<0.0001$, Previous AMI $P<0.0001$.

\section{Collection and analysis of serum and plasma samples}


Blood samples were collected from an antecubital vein from all HF patients and controls. Serum and plasma samples from the HF patients were collected on the enrolment day.

To measure circulating levels of BNP, whole blood was collected in a chilled tube with the anticoagulant EDTA.(14) The sample was promptly put on ice after blood extraction and was centrifuged at RCF $1800 \mathrm{~g}$ for 10 minutes at $4{ }^{\circ} \mathrm{C}$. The supernatant was collected, aliquoted and kept at $-80{ }^{\circ} \mathrm{C}$ until analysis. Quantification of BNP plasma levels was performed and validated in the Biomedical Core Facility of the Hospital Clínic of Barcelona. Briefly, plasma BNP was quantified with a chemiluminometric immunoassay run on the ADVIA Centaur Immunochemistry analyzer (Siemens Healthcare Diagnostics, Tarrytown, NY, USA). The intra-assay coefficient of variation was of 1.8-4.3\% CV and the inter-assay coefficient of variation was of $2.3-4.7 \% \mathrm{CV}$.

Serum samples for sAXL analysis were kept at room temperature for at least 30 minutes after blood extraction, to let the clot to be formed and were later centrifuged at RCF $1800 \mathrm{~g}$ for 10 minutes at room temperature. The supernatant was collected, aliquoted and kept at $-80{ }^{\circ} \mathrm{C}$ until analysis. A new detection method for sAXL quantification was devised in our laboratory using commercially available antibodies. Multiwell plates (96 wells, Rubilabor) were coated with the capture antibodies AF154 (R\&D Systems) at a concentration of $2.0 \mu \mathrm{g} / \mathrm{mL}$ in PBS (137 mM NaCl, $2.7 \mathrm{mM} \mathrm{KCl}$, $8.1 \mathrm{mM} \mathrm{Na}_{2} \mathrm{HPO}_{4}, 1.5 \mathrm{mM} \mathrm{KH} \mathrm{PO}_{4}, \mathrm{pH} 7.2$, filtered through a $0.2 \mu \mathrm{m}$ pore). The plates were sealed and left overnight at room temperature. The plates were washed three times with wash buffer (PBS with $0.05 \%$ Tween20) and then were blocked for 1 hour at room temperature with PBS containing 1\% BSA (Sigma). Serum samples were diluted 200 times in PBS containing 1\% BSA. A standard curve was made by serial dilution of a 
purified sAXL protein produced by recombinant expression of the extracellular part of AXL (R\&D Systems) and concentrations are given as $\mathrm{ng} / \mathrm{mL}$. A negative control without plasma sample was used and pooled-plasma samples were used in each plate as plate controls. After sample addition, the plates were washed three times with wash buffer (PBS with $0.05 \%$ Tween20). Then, $100 \mu \mathrm{L}$ of the BAF154, diluted in PBS containing $1 \%$ BSA was added at a concentration of $50 \mathrm{ng} / \mathrm{mL}$ and incubated 2 hours at room temperature. The aspiration/wash steps were repeated and $100 \mu \mathrm{L}$ of the working dilution of Streptavidin-HRP (Sigma) were added to each well. The plate was covered and incubated for 20 minutes at room temperature. The aspiration/wash steps were repeated and a solution of tetramethyl-benzidine (slow kinetic form; Sigma) was added to the well. The reaction was stopped with sulfuric acid at $1 \mathrm{M}$ concentration. The absorbance of wells was determined with an automatic plate reader at $450 \mathrm{~nm}$. Intraassay variability was determined by evaluating eight replicates of two standard samples. The mean \%CVs was 6.45 and 9.21, respectively. Inter-assay mean \%CV was determined in six different plates and gave the value of 8.32 .

\section{Data analysis}

Logarithmic transformation with base e (ln) was applied to sAXL and BNP values to achieve a normal distribution. $\mathrm{Ln}(\mathrm{sAXL})$ and $\mathrm{Ln}(\mathrm{BNP})$ were back transformed and results are discussed in the text with the original sAXL and BNP values. The independent samples T-test was applied to analyze for $\ln (\mathrm{sAXL})$ differences between the control and the patients groups or between two groups of patients. Patients were stratified as patients with low sAXL levels (L-sAXL; sAXL values below the $3^{\text {rd }}$ quartile $98.1 \mathrm{ng} / \mathrm{mL})$ or high sAXL (H-sAXL; sAXL values equal or above the $3^{\text {rd }}$ quartile $98.1 \mathrm{ng} / \mathrm{mL}$ ). Differences in continuous variables between both groups were 
assessed with a U Mann-Whitney test and differences in discontinuous variables with a Chi-Square test. Values are given as mean and standard error of the mean (SEM), unless otherwise stated. We analyzed whether the continuous variables that had different mean values in the two HF groups with L-sAXL $<98.1 \mathrm{ng} / \mathrm{mL}$ or $\mathrm{H}-\mathrm{sAXL} \geq 98.1 \mathrm{ng} / \mathrm{mL}$ (Table 1 and Table 2), correlated with $\ln (\mathrm{sAXL})$ serum values with the Pearson correlation factor. The variables that were considered for a putative correlation with $\ln (\mathrm{sAXL})$ serum values were: systolic blood pressure, diastolic blood pressure, 6-minutes walk distance, serum creatinine levels, glomerular filtration rate, $\mathrm{C}$ reactive protein, uric acid, hemoglobin, hematocrit and hematies and lymphocyte count. We also assessed whether there was a correlation between the sAXL serum values and the established HF biomarker BNP. All variables correlated with $\ln (\mathrm{sAXL})$ serum values and were introduced in the linear regression model as independent variables and $\ln (\mathrm{sAXL})$ serum levels as the dependant variable. ENTER and STEPWISE methods were both used for introducing the independent variables.

The relationship between $\ln (\mathrm{sAXL})$ and the non-continuous variables that were different between the two HF groups (sAXL $<98.1 \mathrm{ng} / \mathrm{mL}$ or $\mathrm{sAXL} \geq 98.1 \mathrm{ng} / \mathrm{mL}$, was evaluated by an unianova analysis. The variables valvular etiology and congestion signs did not present statistical differences in the unianova analysis and were not further studied. The variables dyslipidemia, NYHA classification and atrial fibrillation showed differences with the unianova analysis and were introduced in the linear regression model together with the continuous variables serum creatinine levels and systolic blood pressure. Receiver-Operator Characteristic Curve (ROC) analysis of sAXL for discriminating HF patients from controls was performed. The cut-off value for calculation of the specificity and sensitivity was the nearest point to the upper-left corner and the point with highest Youden's index value. 
HF evolution measured by time to any event or to the set of events all-cause mortality, heart transplantation and hospitalizations due to HF was analysed using statistical survival techniques. The $3^{\text {rd }}$ quartile value of the sAXL distribution $(98.1 \mathrm{ng} / \mathrm{mL})$ or of the BNP distribution $(362.7 \mathrm{pg} / \mathrm{mL})$ were used as cut-off points for stratification in the Kaplan-Meier survival curves, and patients were divided in three groups with sAXL above the $3^{\text {rd }}$ quartile, with BNP above the $3^{\text {rd }}$ quartile or with both, sAXL and BNP, above the $3^{\text {rd }}$ quartile. Patients lost at the follow-up (10 out of 192) were not included in the analysis. Univariate Cox regression model was evaluated with either all events or with the three HF events; all-cause mortality, heart transplantation or HF hospitalization or with only the two major events all-cause mortality, heart transplantation as outcomes. Hazard ratios were also calculated with the Cox proportional hazard analysis. A Cox regression analysis with a forward stepwise model was performed to verify whether the variable of sAXL values belonging to the $3^{\text {rd }}$ quartile added prognostic value to the evaluation of patients with BNP levels belonging to the $3^{\text {rd }}$ quartile.

All $\mathrm{P}$ values reported are 2 sided, and those that were less than 0.05 were considered to be statistically significant. Statistics were calculated with the SPSS version 18.0 (SPSS Inc. Chicago, Illinois).

\section{Results}

AXL protein is higher in myocardial samples from end-stage HF patients than in controls

Western blot analysis of myocardial biopsies from 26 individuals showed the presence of AXL as two high molecular weight bands of approximately 160, $140 \mathrm{kDa}$ (Figure 1A), and three other bands of lower molecular weight $(120,75$ and $50 \mathrm{kDa})$, possibly corresponding to proteolytic fragments or splicing variants of the full length 
membrane receptor. The upper 160 and $140 \mathrm{kDa}$ bands were quantified by densitometry and compared, showing that samples from $15 \mathrm{HF}$ patients undergoing heart transplantation had a 6-fold increase in AXL immunoreactivity compared to 11 control donors with healthy hearts $(1.2 \pm 0.2$ Relative Units (RU) versus $0.2 \pm 0.5 \mathrm{RU} ; P<0.0001$, Figure 1B). ADAM10? Correlació AXL I ADAM10?

\section{sAXL is higher in HF patients than in controls and correlates with parameters associated with the progression of $\mathrm{HF}$}

sAXL levels in serum were higher in patients $86.3 \pm 2.0 \mathrm{ng} / \mathrm{mL}$ than in controls $67.8 \pm 2.0 \mathrm{ng} / \mathrm{mL}(P<0.0001$, Figure $2 \mathrm{~A})$. Higher sAXL values were also encountered in patients with NYHA class III-IV 100.2 $\pm 4.9 \mathrm{ng} / \mathrm{mL}(\mathrm{n}=54)$ compared to class II patients $80.9 \pm 1.9 \mathrm{ng} / \mathrm{ml}(\mathrm{n}=138 ; P=0.0001$, Figure $2 \mathrm{~B})$. Similar sAXL values were found in HF patients with different etiologies, with the exception of higher values in patients with valvular etiology, when compared against all other HF patients (table 1 and Figure 3).

Receiver-Operator Characteristic Curve (ROC) analysis of sAXL for discriminating HF patients from controls was performed. The area under the curve (AUC) was 0.715 and its $95 \%$ confidence interval estimate was from 0.649 to 0.782 $(P<0.0001)$. A cut-off point value of $71 \mathrm{ng} / \mathrm{ml}$ of sAXL gave a $70.3 \%$ of sensitivity and $64.2 \%$ of specificity.

\section{Patients' characteristics}

Comparison of the demographic and clinical characteristics of $\mathrm{HF}$ patients classified as patients with serum sAXL levels lower than $98.1 \mathrm{ng} / \mathrm{mL}$, the $3^{\text {rd }}$ quartile value of sAXL (L-sAXL, $n=144$ ) or patients with higher or equal serum sAXL levels than $98.1 \mathrm{ng} / \mathrm{mL}(\mathrm{H}-\mathrm{sAXL}, \mathrm{n}=48)$ is summarized in Tables 1 to 4 . Lower systolic 
arterial pressure $(P<0.005)$ and lower diastolic arterial pressure $(P<0.01)$ and less functional capacity measured with the 6-minutes walk test $(P<0.05)$ were found in the H-sAXL group when compared to the L-sAXL group (Table 1). Among common cardiovascular risk factors, lower proportion of dyslipidemia were found in the H-sAXL than in the L-sAXL group $(P<0.005$, Table 1$)$. When comparing the frequency of symptoms and signs between both groups, we found a higher proportion of patients with congestion signs, in the H-sAXL than in the L-sAXL group $(P<0.01$, Table 2$)$.

No differences in the echocardiographic characteristics were found between both groups of HF (Table 2). When comparing ECG findings, we encountered that the HsAXL group had a higher percentage of patients with atrial fibrillation than the L-sAXL group $(P<0.05$, Table 2$)$. Patients in the H-sAXL group had higher values of BNP $(P=0.001)$, serum creatinine $(P<0.001)$, lower glomerular filtration rate $(P<0.0001)$, higher uric acid levels $(P<0.05)$ and higher $\mathrm{C}$ Reactive Protein $(P<0.01$, Table 3$)$. Analysis of blood parameters showed that the H-sAXL group had lower lymphocytes $(P<0.05)$ and erythrocyte count $(P=0.001)$, hemoglobin levels $(P<0.05)$ and hematocrit $(P<0.05$, Table 3$)$. No other differences in laboratory values were found between the two subsets of patients. Pharmacological treatment was very similar in both groups except for a lower intake of statins in the H-sAXL group $(P<0.01$, Table 4$)$ and for a higher proportion of patients taking diuretics, oral digoxin and antiarrhytmics in this group that almost reached significant differences.

\section{Analysis of SAXL with other clinical variables}

We next analyzed whether the continuous variables that had different mean values in the two HF groups with $\mathrm{L}-\mathrm{sAXL}<98.1 \mathrm{ng} / \mathrm{mL}$ or $\mathrm{H}-\mathrm{sAXL} \geq 98.1 \mathrm{ng} / \mathrm{mL}$ 
(Tables 1 to 3 ), correlated with $\ln (\mathrm{sAXL})$ serum values with the Pearson correlation factor. We found that $\ln (\mathrm{sAXL})$ serum values directly correlated with $\ln (\mathrm{BNP})$ $(\mathrm{R}=0.270, P<0.001, \mathrm{n}=167)$ serum creatinine levels $(\mathrm{R}=0.36, P<0.0001, \mathrm{n}=189), \mathrm{C}$ reactive protein $(\mathrm{R}=0.227, P<0.01, \mathrm{n}=143)$, uric acid $(\mathrm{R}=0.253, P<0.01, \mathrm{n}=132)$ and inversely with systolic blood pressure $(\mathrm{R}=-0.323, P<0.0001, \mathrm{n}=184)$ diastolic blood pressure $(\mathrm{R}=-0.238, P=0.001, \mathrm{n}=183), 6$-minutes walk distance $(\mathrm{R}=-0.207, P<0.01$, $\mathrm{n}=171)$, glomerular filtration rate $(\mathrm{R}=-0.296, P<0.0001, \mathrm{n}=178)$, hemoglobin $(\mathrm{R}=-0.217$, $P<0.01, \mathrm{n}=187)$, hematocrit $(\mathrm{R}=-0.207, P<0.01, \mathrm{n}=189)$, hematies count $(\mathrm{R}=-0.243$, $P<0.01, \mathrm{n}=183)$ and lymphocytes count $(\mathrm{R}=-0.211, P<0.01, \mathrm{n}=188)$.

Evaluation of the putative interaction between $\ln (\mathrm{sAXL})$ and the continuous variables that correlated with $\ln (\mathrm{sAXL})$ was done with a linear regression method with $\ln (\mathrm{sAXL})$ as a dependant variable. We found that serum creatinine levels $(\mathrm{P}<0.0001)$ and systolic blood pressure $(\mathrm{P}<0.001)$ were predictive of $\ln (\mathrm{s} A X L)$ serum levels. And all other continuous variables, including BNP, were not predictive. When we introduced non-continuous variables in the linear regression model atrial fibrillation was predictive of $\ln (\mathrm{sAXL})(\mathrm{P}<0.001)$ together with serum creatinine levels and systolic blood pressure.

We also analyzed whether sAXL levels correlated with variables such as age, gender, and risk factors in controls. Higher sAXL values were quantified in female controls $(73.2 \pm 3.5 \mathrm{ng} / \mathrm{mL}, \mathrm{n}=36)$ than in male controls $(64.5 \pm 3.3 \mathrm{ng} / \mathrm{mL}, \mathrm{n}=31$, ) but the difference was not statistically different. No differences of sAXL levels were found with controls age, and common cardiovascular risk factors (data not shown).

\section{Analysis of SAXL in one year follow-up events}


At one year +/- 1 month after enrollment 52 patients out of $182(29 \%)$ had had at least one event of any kind and the remaining 130 patients did not have any event (71\%). When considering only major HF events (all-cause mortality, cardiac transplantation or HF hospitalizations), 33 patients had an event (18\%) and 149 (82\%) did not have any HF event. Kaplan-Meier survival curves and Cox regression analysis showed time course differences in patients that suffered from major HF events between patients with SAXL values lower or higher than the cut-off point of the $3^{\text {rd }}$ quartile $98.1 \mathrm{ng} / \mathrm{mL}$ $(P<0.001$, figure $4 \mathrm{~A})$. A $35 \%$ of patients with sAXL values higher than $98.1 \mathrm{ng} / \mathrm{mL}$ had suffered one of these events one year after enrollment, and their hazard ratio (HR) was 3.31. Such differences were also observed between patients with BNP values lower or higher than the cut-off point of the $3^{\text {rd }}$ quartile $362.7 \mathrm{pg} / \mathrm{mL}(P<0.0001$, figure 4B). A total of $42 \%$ of patients with BNP values higher than $362.7 \mathrm{pg} / \mathrm{mL}$ presented a major HF event at one year follow-up, and the HR of these patients was 4.4. When we classified patients with a combination of both, sAXL and BNP, values above the $3^{\text {rd }}$ quartile or not, we found higher differences in the Cox regression model $(P<0.0001$, figure 4B) than when considering sAXL or BNP values alone. In this case, the percentage of patients in the high sAXL and BNP group that had undergone all-cause mortality, transplantation or $\mathrm{HF}$ hospitalizations rose to $62 \%$ and the HR to 6.77 . Furthermore, a Cox regression analysis with a forward stepwise model showed that the addition of the variable of patients belonging to the sAXL $3^{\text {rd }}$ quartile added prognostic value for major $\mathrm{HF}$ events to the variable of patients belonging to the BNP $3^{\text {rd }}$ quartile $(\mathrm{p}<0.05)$.

Remarkably, when considering only the major events all-cause mortality and heart transplantation, Kaplan-Meier curves and Cox regression analysis with patients with high sAXL also showed differences when compared to patients with low sAXL 
values $(P<0.001, \mathrm{HR}=4.40)$. Those differences were also found when analyzing patients according to their BNP levels $(P<0.001, \mathrm{HR}=5.14)$ and were higher when comparing patients with both high sAXL and BNP levels and the rest $(P<0.0001, \mathrm{HR}=8.54)$.

Kaplan-Meier curves and Cox regression analysis considering all types of events together also presented differences between patients with high and low sAXL $(P<0.05$, $H R=1.85$ ) but the differences were less than when considering only the major HF events or only all-cause mortality and heart transplantation. The survival curves with all events were also different for patients with BNP values above the $3^{\text {rd }}$ quartile $(P<0.001$, $\mathrm{HR}=3.04)$ or with both BNP and sAXL values above the $3^{\text {rd }}$ quartile $(P<0.001$, $\mathrm{HR}=4.13)$.

\section{Discussion}

In this report we demonstrate for the first time that $\mathrm{AXL}$ is expressed in the heart and that is increased in myocardial tissue from HF patients compared to healthy hearts and, in accordance with this over-expression, the serum concentration of sAXL is higher in HF patients than in controls. Furthermore, sAXL levels increase in aggravated $\mathrm{HF}$, in patients with functional class III-IV and with multiple clinical parameters that indicate a worse HF prognosis but no correlation with echocardiographic parameters was found. Also, linear regression analysis showed that BNP levels did not predict sAXL levels, suggesting that both proteins are elevated through independent mechanisms.

High sAXL values at the enrollment time were related to the HF events all-cause mortality, transplantation or HF hospitalizations at one year follow-up and it added predictive value to high BNP levels. All together these results, suggest that sAXL can be a novel player of the HF pathophysiology, acting in a BNP independent pathway. 
The detection of AXL protein in myocardial biopsies confirms results in silico that showed a relatively high expression of $A X L$ probes in heart and cardiomyocytes (Gene atlas, human probe 202686_s_at).(10) Moreover, it suggests that at least part of the soluble receptor detected in serum could be originated in heart after specific proteolytic shedding, as has been shown in other cell types.(12) ADAM10?

Currently, we do not know the role of AXL in HF and whether its expression is deleterious for the heart or if it is a compensatory mechanism. The GAS6/AXL interaction has demonstrated a prominent role in vascular physiology, including biological processes implicated in HF such as inflammation, tissue remodeling, vascular calcification and atherosclerosis. $(15,16)$ Tjwa et al(17) showed that GAS6 facilitates the interactions between endothelial cells, platelets, and leukocytes under inflammatory conditions. This mechanism is likely mediated by AXL, as AXL knockout mice present lower extravasated cells after vascular injury.(18) AXL also appears to have an important role in the vascular response to different injuries, including mechanical damage and hypertension-associated vascular remodeling.(18-21) A third process where AXL plays a prominent role is vascular calcification. AXL activation prevents the osteogenic differentiation of pericytes and vascular smooth muscle phosphate induced calcification. $(22,23)$ In addition, Son et al described the GAS6/AXL pathway as a central interface for the protective effects of statins on vascular smooth muscle calcification.(24) Finally, AXL has also been found to have a role in atherosclerosis.(8, 9) Our results showed correlation of sAXL levels with the inflammatory parameter Creactive protein (CRP) (25) in HF patients. On the other hand, we find a higher proportion of dyslipidemia in patients with lower values of sAXL, which would not sustain a role of AXL in the atherosclerosis process in this group of patients with HF. 
Experimentation with animal models is needed in order to ascertain whether there is a causative relation of AXL and any of these pathways in HF.

The lack of correlation of sAXL circulating levels and echocardiographic parameters, such as LVEDD, LVESD and LVEF, suggests that AXL shedding into blood follows different triggering signals than BNP. Natriuretic peptides are shed from the heart when there is an overload on any chamber of the heart, and hence there is a stretch situation $(26,27)$. It should be emphasized that even though $\ln B N P$ and $\ln$ AXL values correlate; BNP does not appear to be a predictor of sAXL levels in a linear regression model.

Circulating sAXL in patients and controls correlates with several parameters that are altered in HF and that lead to a worse prognosis. In our results, higher sAXL levels are found in HF patients with more functional limitation determined either by the NYHA classification or by the formal 6MWT of exercise tolerance. $(28,29)$ Also, HF patients with higher peripheral sAXL levels presented lower systolic and diastolic blood pressure. Low arterial pressure will compromise critical organ perfusion and is associated with a poorer prognosis.(30) Furthermore, we quantified increased serum sAXL levels together with increased kidney dysfunction determined by either higher serum creatinine levels or by lower glomerular filtration rates. Indeed, the GAS6/AXL pathway has been shown to be implicated in development of several renal pathologies. $(31,32)$ Also, HF patients in the H-sAXL group had higher uric acid levels and sAXL was highly correlated with bilirubin and anemia in HF patients, three parameters that are associated with adverse outcomes in HF.(33-35)

Our ROC analysis of sAXL for discriminating HF patients from controls, shows that SAXL as a putative diagnostic marker has much less sensitivity and specificity than BNP (data not shown). In the acute setting, testing for the natriuretic biomarkers to rule 
out or to confirm the HF diagnosis is well established(28, 36, 37) and are indicated in Clinical Guidelines. $(2,29,38)$ The natriuretic peptides are also well established HF prognostic markers. The higher rate of HF events we find in this group of HF patients with reduced ejection fraction and high BNP levels is in accordance with other results in the literature that have shown that the natriuretic peptides are powerful predictors of adverse outcomes in patients with $\operatorname{HF}(39-44)$.

Other biomarkers such as cardiac necrosis markers, troponin I or T, $(45,46)$ or fibrosis markers such as soluble ST2 (47-49) and galectin-3 $(50,51)$ have been shown to be predictive of hospitalization and death in patients with HF and have an additive prognostic value to natriuretic peptide levels. The carbohydrate antigen 125 (CA125) also has a predictive value of adverse outcomes in HF patients (52) and adds prognostic value to $\mathrm{BNP}$ (53). Our results indicate that $\mathrm{SAXL}$ is also predictive of HF events at short-term follow-up and that the combination of an elevated sAXL with BNP was a better predictor of major HF events and of all-cause mortality and heart transplantation than either of the two markers alone.

The fact that AXL is 6-fold over expressed in pathological ventricular heart biopsies, suggests that the increased levels encountered in the peripheral blood samples from HF patients is shed from the heart.

\section{Conclusions}

Altogether, we have presented in this report quantification of AXL in heart biopsies by western blot and of sAXL in serum by a new enzyme-linked immunosorbent assay (ELISA). sAXL levels in HF correlate with BNP levels, the current marker of HF and with other clinical parameters related to aggravated HF. Remarkably, high sAXL levels predicted HF events at short term follow-up, and added 
predictive value to BNP. Based on these results, we conclude that sAXL appears to have a role in HF progression in a distinct molecular pathway than BNP.

\section{Study limitations}

The control group of the sAXL serum analysis was not matched with the HF group in age and cardiovascular risk factors. This is a serious limitation of this study, but analysis of sAXL levels in the control group indicated that was independent of the subject's age and sex. On the other hand, the difficulty of finding controls with several cardiovascular risk factors and no cardiovascular disease precluded the obtention of such samples.

This is a two center study from the same metropolitan area, with consecutive patients enrolled. And although it has some advantages, such as including a more homogeneous diagnosis, demographics and laboratory techniques, validation of these results in other centers or in multicentric studies will be needed to confirm the increase of sAXL in HF patients from other populations.

We were not able to obtain the cause of mortality from the records in most of the cases. Therefore, we have analyzed the variable all-cause mortality and could not do analysis with only cardiovascular causes of mortality.

\section{Acknowledgments}

We want to thank the staff for their research support, specially Natalia Pérez, Nadia Castillo, Vanesa Hervás, Silvia Vidorreta and Magda Castillo of the Institut Clínic de Malalties Cardiovasculars of Hospital Clinic of Barcelona; Teresa Martorell of Hospital Clinic of Barcelona; Anghara Menéndez, from IIBB-CSIC; and Maite Domingo and Teresa Torrades of the Cardiology Department of the Hospital Sant Pau, for technical 
help, data collection and/or patient management, and Eduard Guasch for helpful discussion of the results.

Reference List

1. Heidenreich PA, Trogdon JG, Khavjou OA et al. Forecasting the future of cardiovascular disease in the United States: a policy statement from the American Heart Association. Circulation 2011 Mar 1;123(8):933-944.

2. McMurray JJ, Adamopoulos S, Anker SD et al. ESC guidelines for the diagnosis and treatment of acute and chronic heart failure 2012: The Task Force for the Diagnosis and Treatment of Acute and Chronic Heart Failure 2012 of the European Society of Cardiology. Developed in collaboration with the Heart Failure Association (HFA) of the ESC. Eur J Heart Fail 2012 Aug;14(8):803-869.

3. The survival of patients with heart failure with preserved or reduced left ventricular ejection fraction: an individual patient data meta-analysis. Eur Heart J 2012 Jul;33(14):1750-1757.

4. Mudd JO, Kass DA. Tackling heart failure in the twenty-first century. Nature 2008 Feb 21;451(7181):919-928.

5. Whelan RS, Kaplinskiy V, Kitsis RN. Cell death in the pathogenesis of heart disease: mechanisms and significance. Annu Rev Physiol 2010;72:19-44. 
6. Bellido-Martin L, de Frutos PG. Vitamin K-dependent actions of Gas6. Vitam Horm 2008;78:185-209.

7. Evenas P, Garcia de FP, Nicolaes GA, Dahlback B. The second laminin G-type domain of protein $\mathrm{S}$ is indispensable for expression of full cofactor activity in activated protein C-catalysed inactivation of factor Va and factor VIIIa. Thromb Haemost 2000 Aug;84(2):271-277.

8. Clauser S, Meilhac O, Bieche I et al. Increased secretion of Gas6 by smooth muscle cells in human atherosclerotic carotid plaques. Thromb Haemost 2012 Jan;107(1):140-149.

9. Hurtado B, Munoz X, Recarte-Pelz P et al. Expression of the vitamin K-dependent proteins GAS6 and protein S and the TAM receptor tyrosine kinases in human atherosclerotic carotid plaques. Thromb Haemost 2011 May;105(5):873-882.

10. Su AI, Wiltshire T, Batalov S et al. A gene atlas of the mouse and human proteinencoding transcriptomes. Proc Natl Acad Sci U S A 2004 Apr 20;101(16):60626067.

11. Ekman C, Stenhoff J, Dahlback B. Gas6 is complexed to the soluble tyrosine kinase receptor Axl in human blood. J Thromb Haemost 2010 Apr;8(4):838-844.

12. Scutera S, Fraone T, Musso T et al. Survival and migration of human dendritic cells are regulated by an IFN-alpha-inducible Axl/Gas6 pathway. J Immunol 2009 Sep 1;183(5):3004-3013.

13. Batlle M, Roig E, Perez-Villa F et al. Increased expression of the reninangiotensin system and mast cell density but not of angiotensin-converting 
enzyme II in late stages of human heart failure. J Heart Lung Transplant 2006 Sep;25(9):1117-1125.

14. Casals G, Azzalini L, Tomas C et al. Admission B-type natriuretic peptide retains prognostic value in patients with acute coronary syndrome and preserved left ventricular ejection fraction. Int J Cardiol 2012 Jul 26;158(3):459-460.

15. Fernandez-Fernandez L, Bellido-Martin L, Garcia dF. Growth arrest-specific gene 6 (GAS6). An outline of its role in haemostasis and inflammation. Thromb Haemost 2008 Oct;100(4):604-610.

16. Laurance S, Lemarie CA, Blostein MD. Growth arrest-specific gene 6 (gas6) and vascular hemostasis. Adv Nutr 2012 Mar;3(2):196-203.

17. Tjwa M, Bellido-Martin L, Lin Y et al. Gas6 promotes inflammation by enhancing interactions between endothelial cells, platelets, and leukocytes. Blood 2008 Apr 15;111(8):4096-4105.

18. Korshunov VA, Mohan AM, Georger MA, Berk BC. Axl, a receptor tyrosine kinase, mediates flow-induced vascular remodeling. Circ Res 2006 Jun 9;98(11):1446-1452.

19. Gerloff J, Korshunov VA. Immune modulation of vascular resident cells by Axl orchestrates carotid intima-media thickening. Am J Pathol 2012 May;180(5):2134-2143.

20. Konishi A, Aizawa T, Mohan A, Korshunov VA, Berk BC. Hydrogen peroxide activates the Gas6-Axl pathway in vascular smooth muscle cells. J Biol Chem 2004 Jul 2;279(27):28766-28770. 
21. Korshunov VA, Daul M, Massett MP, Berk BC. Axl mediates vascular remodeling induced by deoxycorticosterone acetate-salt hypertension. Hypertension 2007 Dec;50(6):1057-1062.

22. Collett G, Wood A, Alexander MY et al. Receptor tyrosine kinase Axl modulates the osteogenic differentiation of pericytes. Circ Res 2003 May 30;92(10):11231129.

23. Collett GD, Sage AP, Kirton JP, Alexander MY, Gilmore AP, Canfield AE. Axl/phosphatidylinositol 3-kinase signaling inhibits mineral deposition by vascular smooth muscle cells. Circ Res 2007 Mar 2;100(4):502-509.

24. Son BK, Kozaki K, Iijima K et al. Statins protect human aortic smooth muscle cells from inorganic phosphate-induced calcification by restoring Gas6-Axl survival pathway. Circ Res 2006 Apr 28;98(8):1024-1031.

25. Braunwald E. Biomarkers in heart failure. N Engl J Med 2008 May 15;358(20):2148-2159.

26. Wiese S, Breyer T, Dragu A et al. Gene expression of brain natriuretic peptide in isolated atrial and ventricular human myocardium: influence of angiotensin II and diastolic fiber length. Circulation 2000 Dec 19;102(25):3074-3079.

27. Yasue $\mathrm{H}$, Yoshimura M, Sumida $\mathrm{H}$ et al. Localization and mechanism of secretion of B-type natriuretic peptide in comparison with those of A-type natriuretic peptide in normal subjects and patients with heart failure. Circulation 1994 Jul;90(1):195-203. 
28. Maisel AS, Krishnaswamy P, Nowak RM et al. Rapid measurement of B-type natriuretic peptide in the emergency diagnosis of heart failure. N Engl J Med 2002 Jul 18;347(3):161-167.

29. Yancy CW, Jessup M, Bozkurt B et al. 2013 ACCF/AHA Guideline for the Management of Heart Failure: A Report of the American College of Cardiology Foundation/American Heart Association Task Force on Practice Guidelines. J Am Coll Cardiol 2013 Jun 5.

30. Pocock SJ, Ariti CA, McMurray JJ et al. Predicting survival in heart failure: a risk score based on 39372 patients from 30 studies. Eur Heart J 2013 May;34(19):1404-1413.

31. Lee IJ, Hilliard B, Swami A et al. Growth arrest-specific gene 6 (Gas6) levels are elevated in patients with chronic renal failure. Nephrol Dial Transplant 2012 Aug 20.

32. Yanagita M. Gas6, warfarin, and kidney diseases. Clin Exp Nephrol 2004 Dec;8(4):304-309.

33. Ambrosy AP, Vaduganathan M, Huffman MD et al. Clinical course and predictive value of liver function tests in patients hospitalized for worsening heart failure with reduced ejection fraction: an analysis of the EVEREST trial. Eur J Heart Fail 2012 Mar;14(3):302-311.

34. Tang YD, Katz SD. Anemia in chronic heart failure: prevalence, etiology, clinical correlates, and treatment options. Circulation 2006 May 23;113(20):2454-2461. 
35. Anker SD, Doehner W, Rauchhaus M et al. Uric acid and survival in chronic heart failure: validation and application in metabolic, functional, and hemodynamic staging. Circulation 2003 Apr 22;107(15):1991-1997.

36. Davis M, Espiner E, Richards G et al. Plasma brain natriuretic peptide in assessment of acute dyspnoea. Lancet 1994 Feb 19;343(8895):440-444.

37. Januzzi JL, van Kimmenade R, Lainchbury $\mathbf{J}$ et al. NT-proBNP testing for diagnosis and short-term prognosis in acute destabilized heart failure: an international pooled analysis of 1256 patients: the International Collaborative of NT-proBNP Study. Eur Heart J 2006 Feb;27(3):330-337.

38. Tang WH, Francis GS, Morrow DA et al. National Academy of Clinical Biochemistry Laboratory Medicine Practice Guidelines: clinical utilization of cardiac biomarker testing in heart failure. Clin Biochem 2008 Mar;41(4-5):210221.

39. Berger R, Huelsman M, Strecker K et al. B-type natriuretic peptide predicts sudden death in patients with chronic heart failure. Circulation 2002 May 21;105(20):2392-2397.

40. Dini FL, Gabutti A, Passino C, Fontanive P, Emdin M, De Tommasi SM. Atrial fibrillation and amino-terminal pro-brain natriuretic peptide as independent predictors of prognosis in systolic heart failure. Int J Cardiol 2010 Apr $30 ; 140(3): 344-350$.

41. Fonarow GC, Peacock WF, Phillips CO, Givertz MM, Lopatin M. Admission Btype natriuretic peptide levels and in-hospital mortality in acute decompensated heart failure. J Am Coll Cardiol 2007 May 15;49(19):1943-1950. 
42. Koglin J, Pehlivanli S, Schwaiblmair M, Vogeser M, Cremer P, vonScheidt W. Role of brain natriuretic peptide in risk stratification of patients with congestive heart failure. J Am Coll Cardiol 2001 Dec;38(7):1934-1941.

43. Maisel A, Mueller C, Adams K et al. State of the art: using natriuretic peptide levels in clinical practice. Eur J Heart Fail 2008 Sep;10(9):824-839.

44. van Veldhuisen DJ, Linssen GC, Jaarsma T et al. B-type natriuretic peptide and prognosis in heart failure patients with preserved and reduced ejection fraction. $\mathrm{J}$ Am Coll Cardiol 2013 Apr 9;61(14):1498-1506.

45. Jungbauer CG, Riedlinger J, Buchner $\mathrm{S}$ et al. High-sensitive troponin $\mathrm{T}$ in chronic heart failure correlates with severity of symptoms, left ventricular dysfunction and prognosis independently from $\mathrm{N}$-terminal pro-b-type natriuretic peptide. Clin Chem Lab Med 2011 Nov;49(11):1899-1906.

46. Zairis MN, Tsiaousis GZ, Georgilas AT et al. Multimarker strategy for the prediction of 31 days cardiac death in patients with acutely decompensated chronic heart failure. Int J Cardiol 2010 Jun 11;141(3):284-290.

47. Pascual-Figal DA, Manzano-Fernandez S, Boronat M et al. Soluble ST2, highsensitivity troponin $\mathrm{T}$ - and $\mathrm{N}$-terminal pro-B-type natriuretic peptide: complementary role for risk stratification in acutely decompensated heart failure. Eur J Heart Fail 2011 Jul;13(7):718-725.

48. Weinberg EO, Shimpo M, Hurwitz S, Tominaga S, Rouleau JL, Lee RT. Identification of serum soluble ST2 receptor as a novel heart failure biomarker. Circulation 2003 Feb 11;107(5):721-726. 
49. Bayes-Genis A, de AM, Galan A, Sanz H et al. Combined use of high-sensitivity ST2 and NTproBNP to improve the prediction of death in heart failure. Eur J Heart Fail 2012 Jan;14(1):32-38.

50. Lok DJ, van der MP, de la Porte PW et al. Prognostic value of galectin-3, a novel marker of fibrosis, in patients with chronic heart failure: data from the DEAL-HF study. Clin Res Cardiol 2010 May;99(5):323-328.

51. van Kimmenade RR, Januzzi JL, Jr., Ellinor PT et al. Utility of amino-terminal pro-brain natriuretic peptide, galectin-3, and apelin for the evaluation of patients with acute heart failure. J Am Coll Cardiol 2006 Sep 19;48(6):1217-1224.

52. Monteiro S, Franco F, Costa S et al. Prognostic value of CA125 in advanced heart failure patients. Int J Cardiol 2010 Apr 1;140(1):115-118.

53. Nunez J, Sanchis J, Bodi V et al. Improvement in risk stratification with the combination of the tumour marker antigen carbohydrate 125 and brain natriuretic peptide in patients with acute heart failure. Eur Heart J 2010 Jul;31(14):17521763.

54. Vuoriluoto K, Haugen H, Kiviluoto $\mathrm{S}$ et al. Vimentin regulates EMT induction by Slug and oncogenic H-Ras and migration by governing Axl expression in breast cancer. Oncogene 2011 Mar 24;30(12):1436-1448. 
Table 1. Baseline demographic, prior history and clinical characteristics of HF patients stratified by SAXL $3^{\text {rd }}$ quartile level.

sAXL, ng/mL

Parameter

$<98.1$

$\geq 98.1$

$P$

No of patients

144

48

Demographics

Age, $y$,

$$
61 \pm 1
$$

$64 \pm 1$

ns

Male, n (\%)

$123(85)$

$41(85)$

ns

Female, n (\%)

$21(15)$

$7(15)$

ns

Risk Factors, n (\%)

Hypertension

Dyslipidemia

Diabetes Mellitus

Current/former smoker

Previous AMI

Clinical characteristics

Body Mass Index, Kg/m2

NYHA classification, $\mathrm{n}(\%)$

II

III-IV
$28.1 \pm 0.4$

$27.9 \pm 0.8$

ns

$112(78) \quad 26(54) \quad<0.005$

32 (22)

$22(46)$

$<0.005$

Etiology, n (\%) 


\begin{tabular}{llll} 
Idiopathic & $40(28)$ & $13(27)$ & $\mathrm{ns}$ \\
Ischemic & $75(52)$ & $18(38)$ & $\mathrm{ns}$ \\
Valvular & $12(8)$ & $11(23)$ & $=0.01$ \\
Hypertensive & $4(3)$ & $1(2)$ & $\mathrm{ns}$ \\
Other & $13(9)$ & $5(10)$ & $\mathrm{ns}$ \\
Heart rate, beats/min & $71.5 \pm 1.2$ & $72.2 \pm 2.1$ & $\mathrm{~ns}$ \\
Systolic blood pressure, $\mathrm{mm} \mathrm{Hg}$ & $120.0 \pm 1.9$ & $108.9 \pm 2.4$ & $<0.005$ \\
Diastolic blood pressure, $\mathrm{mm} \mathrm{Hg}$ & $73.3 \pm 1.0$ & $68.3 \pm 1.5$ & $<0.01$ \\
Pulse pressure & $47.1 \pm 1.6$ & $40.6 \pm 2.2$ & $\mathrm{~ns}$ \\
Abdominal perimeter, cm & $102.8 \pm 1.2$ & $103.9 \pm 3.1$ & $\mathrm{~ns}$ \\
6-min walk distance, $\mathrm{m}$ & $418 \pm 8.9$ & $372 \pm 17.4$ & $<0.05$ \\
& & & \\
\hline
\end{tabular}

AMI (acute myocardial infarction) 
Table 2. Baseline HF symptoms and signs, ECG and echocardiographic parameters of HF-REF patients stratified by SAXL levels.

$\mathrm{sAXL}, \mathrm{ng} / \mathrm{mL}$

Parameter

$<98.1$

$\geq 98.1$

$P$

No of patients

144

48

Symptoms and signs

\begin{tabular}{|c|c|c|c|}
\hline Paroxysmal nocturnal dyspnea, n (\%) & $24(18)$ & $6(15)$ & ns \\
\hline Reduction in exercise tolerance, $\mathrm{n}(\%)$ & $56(42)$ & $18(50)$ & $\mathrm{ns}$ \\
\hline Orthopnea, n (\%) & $40(30)$ & $11(28)$ & ns \\
\hline Syncope, n (\%) & $19(14)$ & $4(9)$ & ns \\
\hline Lower extremity edema, n (\%) & $18(13)$ & $6(14)$ & ns \\
\hline Congestion signs, $\mathrm{n}(\%)$ & $4(5)$ & $6(27)$ & $<0.01$ \\
\hline Jugular venous distension, $\mathrm{n}(\%)$ & $7(9)$ & $5(24)$ & ns \\
\hline Hepatojugular reflux, n (\%) & $14(18)$ & $4(20)$ & ns \\
\hline \multicolumn{4}{|l|}{ parameters } \\
\hline Sinusal Rhythm, n (\%) & $86(61)$ & $24(52)$ & ns \\
\hline Atrial Fibrillation, n (\%) & $9(6)$ & $8(17)$ & $<0.05$ \\
\hline QRS length, ms & $134.8 \pm 3.2$ & $134.5 \pm 5.5$ & ns \\
\hline Interval PR, ms & $168.9 \pm 4.0$ & $168.6 \pm 6.0$ & ns \\
\hline Necrosis Q waves, n (\%) & $32(34)$ & $7(24)$ & ns \\
\hline Intervent conduct disorders, $\mathrm{n}(\%)$ & 77 (61) & $28(70)$ & ns \\
\hline
\end{tabular}


Left bundle branch block, n (\%)

$$
18(21)
$$

$8(29)$

ns

Pacemaker, n (\%)

$77(54)$

$29(60)$

ns

Resynchronization therapy, n (\%)

$19(13)$

$4(8)$

ns

Echocardiographic parameters

$\begin{array}{llll}\text { LVESD, mm } & 53.5 \pm 1.0 & 53.6 \pm 1.7 & \mathrm{~ns} \\ \text { LVEDD, mm } & 67.5 \pm 0.8 & 66.9 \pm 1.2 & \mathrm{~ns} \\ \text { LVEF, \% } & 27.6 \pm 0.6 & 26.5 \pm 1.0 & \mathrm{~ns} \\ \text { LAD, mm } & 47.4 \pm 0.7 & 50.5 \pm 1.6 & \mathrm{~ns} \\ \text { IVST, mm } & 10.3 \pm 0.1 & 10.8 \pm 0.3 & \mathrm{~ns} \\ \text { LVPWT, mm } & 9.9 \pm 0.1 & 10.1 \pm 0.2 & \mathrm{~ns} \\ \text { LVH, n }(\%) & 57(42) & 24(53) & \mathrm{ns}\end{array}$

Intervent conduct disorders (Interventricular conduction disorders), LVESD (left ventricle end-systolic diameter), LVEDD (left ventricle end-diastolic diameter), LVEF (left ventricle ejection fraction), LAD (Left atrial diameter), IVST (Interventricular septum thickness), LVPWT (Left ventricular posterior wall thickness), LVH (Left Ventricular Hypertrophy defined as IVST $\geq 11 \mathrm{~mm}$ ). 
Table 3. Baseline laboratory values of HF patients stratified by SAXL levels.

\begin{tabular}{|c|c|c|c|}
\hline \multirow[b]{2}{*}{ Parameter } & \multicolumn{3}{|c|}{$\mathrm{sAXL}, \mathrm{ng} / \mathrm{mL}$} \\
\hline & $<98.1$ & $\geq 98.1$ & $P$ \\
\hline No of patients & 144 & 48 & \\
\hline $\mathrm{BNP}, \mathrm{pg} / \mathrm{mL}$ & $251.9 \pm 37.1$ & $456.6 \pm 69.2$ & $=0.001$ \\
\hline Serum creatinine, $\mathrm{mg} / \mathrm{dL}$ & $1.12 \pm 0.03$ & $1.36 \pm 0.07$ & $<0.001$ \\
\hline Glomerular filtration rate, $\mathrm{mL} / \mathrm{min}$ & $57.9 \pm 0.7$ & $52.2 \pm 1.7$ & $<0.0001$ \\
\hline Sodium, $\mathrm{mEq} / \mathrm{L}$ & $140.0 \pm 0.3$ & $139.3 \pm 0.6$ & ns \\
\hline Potassium, mEq/L & $4.6 \pm 0.04$ & $4.5 \pm 0.09$ & ns \\
\hline Aspartate Aminotransferase, UI/L & $24.4 \pm 0.7$ & $31.9 \pm 5.7$ & ns \\
\hline Alanine Aminotransferase, U/L & $25.6 \pm 1.3$ & $33.6 \pm 8.3$ & ns \\
\hline Bilirubin, mg/dL & $0.80 \pm 0.04$ & $0.88 \pm 0.07$ & ns \\
\hline Uric Acid mg/dL & $6.70 \pm 0.17$ & $7.84 \pm 0.43$ & $<0.05$ \\
\hline Glucose, $\mathrm{mg} / \mathrm{dL}$ & $114.9 \pm 3.1$ & $115.2 \pm 4.3$ & ns \\
\hline Total Cholesterol, mg/dL & $169.0 \pm 3.2$ & $170.8 \pm 5.8$ & ns \\
\hline HDL Cholesterol, mg/dL & $40.9 \pm 0.8$ & $40.1 \pm 1.7$ & ns \\
\hline LDL Cholesterol, mg/dL & $103.1 \pm 2.6$ & $103.8 \pm 5.3$ & ns \\
\hline Triglycerides, mg/dL & $127.3 \pm 5.1$ & $133.8 \pm 12.0$ & ns \\
\hline C Reactive Protein mg/dL & $0.73 \pm 0.16$ & $1.34 \pm 0.64$ & $<0.01$ \\
\hline Thyrotropin, mUI/L & $3.4 \pm 0.7$ & $2.3 \pm 0.3$ & ns \\
\hline Thyroxine, ng/dL & $1.29 \pm 0.02$ & $1.35 \pm 0.06$ & ns \\
\hline Hemoglobin, g/L & $138.3 \pm 1.2$ & $131.5 \pm 2.8$ & $<0.05$ \\
\hline
\end{tabular}


Hematocrit, L/L

Erythrocyte count, 10E12/L

Lymphocytes count, 10E9/L

Platelet count, 10E9/L
$0.423 \pm 0.003 \quad 0.407 \pm 0.008<0.05$

$4.64 \pm 0.04 \quad 4.35 \pm 0.10 \quad=0.001$

$1.9 \pm 0.05 \quad 1.6 \pm 0.08 \quad<0.05$

$222.3 \pm 5.4 \quad 205.0 \pm 8.2 \quad$ ns 
Table 4. Pharmacological treatment of HF patients stratified by SAXL levels.

sAXL, ng/mL

Pharmacological treatment

$<98.1$

$\geq 98.1$

$P$

No of patients

144

48

ACEI, n (\%)

$91(66)$

$34(71)$

ns

$\mathrm{ARB}, \mathrm{n}(\%)$

$36(27)$

$7(15)$

ns

ACEI and/or ARB, n (\%)

$127(91) \quad 41(85)$

ns

Beta-blocker, n (\%)

$132(94) \quad 44(92) \quad n s$

Ca-antagonists, n (\%)

$12(9)$

$3(6)$

ns

Antithrombotic and/or anticoagulant, n (\%)

$110(80)$

$41(85)$

ns

Statins, n (\%)

95 (69)

$21(44)$

$<0.01$

Antidiabetics, n (\%)

$41(30)$

$11(23)$

ns

Diuretics, n (\%)

$104(75)$

$42(88)$

0.055

Antialdosteronic agents, n (\%)

$74(54)$

$28(58)$

ns

Digoxin, n (\%)

$12(9)$

$9(19)$

0.054

Antiarrhythmics, n (\%)

$26(19)$

$15(31)$

0.054

Nitrates, n (\%)

$17(12)$

$8(17)$

ns

Hydralazine, n (\%)

$5(4)$

$3(6)$

ns

Anemia treatment, n (\%)

$6(4)$

$2(4)$

ns

ACEI (Angiotensin Converting Enzyme Inhibitor), ARB (Angiotensin Receptor Blocker). 


\section{Legends}

Figure 1.Western Blot analysis of AXL and ADAM10 expression in myocardial biopsies. A, representative western blot of myocardial biopsies from 2 healthy (control) and 5 pathological (heart failure) left ventricle samples. Thirty $\mu \mathrm{g}$ of protein were loaded in each lane. AXL (upper panel) appears as a doublet of 140-160 kDa bands, while ADAM10 (lower panel) appears as a ... kDa and ....kDa bands. Quantification of the 140 and $160 \mathrm{kDa}$ AXL bands (B) and ... kDa and ....kDa ADAM10 bands (C) by densitometry of 3 different blots with control $(n=11)$ and heart failure $(n=15)$ samples. Results were compared with a T-test $(* * * * P<0.0001)$.

Figure 2. Serum sAXL values from controls and HF patients. A, Boxplot of serum sAXL from controls (C) and heart failure (HF) patients. B, sAXL serum levels from HF patients belonging to NYHA functional class 2 (II) or 3-4 (III-IV). Ln-sAXL between groups was compared with a T-test $(* * * * P<0.0001)$.

Figure 3. Serum sAXL levels from HF patients with different HF etiologies. Boxplot of serum sAXL from HF patients divided in 5 different groups according to their etiology; idiopathic (idiop), ischemic (isch), valvular (valv), hypertensive (hypert) and others.

Figure 4. Kaplan-Meier survival curve from HF patients divided according their sAXL and BNP levels and considering the major HF events (all-cause mortality, transplantation and HF hospitalizations). A, Kaplan-Meier survival curve from patients with sAXL below (L_sAXL, dotted grey line) or above the $3^{\text {rd }}$ quartile cut-off point (98.1 ng/mL, H_sAXL dotted black line). The differences in HF evolution were compared with the Cox Regression model ( $* * * P<0.001)$. B, Kaplan-Meier survival curve from patients with BNP below (L_BNP, dotted grey line) or above the $3^{\text {rd }}$ quartile cut-off point $(362.7$ pg/mL, H_BNP dotted black line, $* * * * P<0.0001)$. Kaplan-Meier 
survival curve from patients with the 2 markers, sAXL and BNP, levels above the $3^{\text {rd }}$ quartiles cut-off points (H_sAXL+BNP black solid line) or with one or both values below the $3^{\text {rd }}$ quartiles cut-off points (L_sAXL+BNP grey solid line, $\left.* * * * P<0.0001\right)$. * $P<0.05$ between H_BNP and H_sAXL+BNP 